\title{
Reduction of FEL Gain Due to Wiggler Errors*
}

\author{
L.H. Yu, S. Krinsky, A. Friedman \\ National Synchrotron Light Source, \\ Brookhaven National Laboratory \\ Upton, NY 11973
}

R.L. Gluckstern and J.B.J. Van Zeijts

Physics Department

University of Maryland

College Park, MD 20742

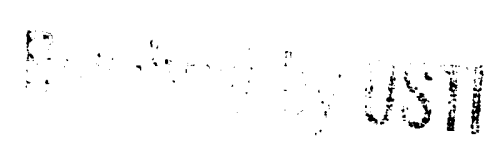

101991

\section{ABSTRACT}

For an FEL operating in the exponential regime before saturation, we consider the effect on the gain of longitudinal velocity variations arising from wiggler field errors. The average gain reduction and the width of the output power distribution are expressed in terms of the mean square average of the ponderomotive phase shift per gain length. A scheme for correcting the electron trajectory using position monitors and dipole correctors is analyzed. Analytic results are compared with numerical simulations. Our work is directly applicable to the design of FEL amplifiers and the results are encouraging for the feasibility of such devices.

\section{DISCLAIMER}

\begin{abstract}
This report was prepared as an account of work sponsored by an agency of the United States Government. Neither the United States Government nor any agency thereof, nor any of their employees, makes any warranty, express or implied, or assumes any legal liability or responsibility for the accuracy, completeness, or usefulness of any information, apparatus, product, or process disclosed, or represents that its use would not infringe privately owned rights. Reference herein to any specific commercial product, process, or service by trade name, trademark, manufacturer, or otherwise does not necessarily constitute or imply its endorsement, recommendation, or favoring by the United States Government or any agency thereof. The views and opinions of authors expressed herein do not necessarily state or reflect those of the United States Government or any agency thereof.
\end{abstract}

* Work performed under the auspices of the U.S. Department of Energy. under contract DE-AC02-76CH00016 


\section{Introduction}

Understanding the effects of wiggler errors $[1-8]$ is of critical importance in the design of free electron lasers. There has been recent work aimed at determining the tolerances which must be imposed on the allowable field errors. Analytic results have been obtained for the effect of wiggler errors on the electron trajectory [7], and for the gain reduction in the low gain regime $e^{[5,6]}$. Computer simulations have been carried out both in the low $[6,8]$ and high gain $[3,4]$ regimes. In a recent paper ${ }^{[9]}$, we have developed an analytic description of the effect of wiggler errors on the gain, for an FEL operating in the exponential regime before saturation, using one-dimensional FEL equations [10-12]. In this paper, we discuss the results of this work, and compare them with numerical simulations.

The effects of wiggler errors can be divided into two classes: (1) Longitudinal velocity fluctuation and drift, which moves the electron beam away from resonance, and (2) transverse trajectory wander which causes the centroid of the electron beam to move away from the radiation beam. We address the longitudinal effects and leave detailed consideration of the mode overlap problem to future work based on a full three-dimensional computer simulation. The determination of the effect of longitudinal velocity variations is dominantly a one-dimensional problem for which we have carried out a detailed analysis [9].

We divide longitudinal velocity effects into two iypes. The first type is wiggling amplitude errors which are correlated over only a few wiggler periods and have no net magnetic field integral. The second type is cumulative steering errors, which produce a drift of the electron trajectory away from the wiggler axis.

For the case of amplitude errors, we find that the required tolerance on the magnetic field fluctuation is relaxed, since it turns out that rather than having to satisfy $(\Delta B / B)_{r m s} \ll \rho$, one only needs to meet $(\Delta B / B)_{r m s}^{2} \ll \rho$, where $\rho$ is the Pierce parameter ${ }^{[10]}$ typically of magnitude $10^{-3}$.

Without correcting the electron trajectory periodically along the wiggler, achievable steering errors will cause a cumulative angular deviation away from the wiggler axis, moving the electron beam away from resonance and, eventually, completely killing the gain. We have derived an expression for the gain reduction when there is trajectory correction and we have found that by installing a sufficient number of correction and monitoring stations 
along the wiggler, the required tolerance on magnetic field steering errors can be relaxed to an achievable limit. The results of our analysis on both type of longitudinal errors are encouraging for the design of single pass FEL amplifiers utilizing long wiggler magnets.

For both types of longitudinal error we have found that the criterion for small gain reduction is determined by keeping the ponderomotive phase shift due to wiggler errors small in one gain length. The ponderomotive phase shift is

$$
\delta=k \int \frac{d z}{v_{0}}\left[v_{\|}\left(\gamma_{0}, z\right)-v_{0}\right]
$$

for a monoenergetic electron beam of energy $\gamma_{0}$, where $v_{0}$ is the average longitudinal velocity in the ideal wiggler, $k$ the radiation wavenumber, and $v_{\|}\left(\gamma_{0}, z\right)$ is the longitudinal velocity in the presence of wiggler errors.

The importance of the ponderomotive phase shift in determining the gain reduction due to wiggler errors has been discussed in refs. [5] and [6] for the low gain regime. In recent work [9], we have considered the high gain regime and explicitly expressed the gain reduction due to wiggler errors in terms of $W$, the average value of the square of the ponderomotive phase shift per gain length which is defined by

$$
W \equiv \frac{1}{T} \overline{\left[\int_{0}^{T} d \tau \frac{d \delta(\tau)}{d \tau}\right]^{2}}
$$

where $\tau=2 \rho k_{w} z$ is the scaled longitudinal coordinate ${ }^{[10]}$ which changes by $2 / \sqrt{3}$ in a gain length, and the average is over an ensemble of wiggler errors. The power growth of the radiation field is proportional to

$$
e^{\left(\sqrt{3}-\frac{2 W}{9}\right) \tau}
$$

In the following sections, we first briefly describe the general results in Section 2 . describing the FEL gain equation with wiggler errors. expressing the output power and the width of its distribution in terms of $W$. Next, in Section 3, we discuss the effect of the wiggling amplitude errors. Then, in Section 4, we describe the effect of steering errors when a scheme for correcting the electron trajectory using position monitors and dipole 
correctors is taken into account. Finally, in Section 5, we address the effect of steering errors when there is no trajectory correction.

\section{General Results on Gain Reduction due to Wiggler Errors}

We will now present the FEL gain equation with wiggler errors. For a detailed derivation we refer to reference [9]. We consider a plane polarized radiation field

$$
E_{x}(z, t)=\frac{1}{2} E(z) e^{i k(z-c t)}+c . c .
$$

where $k$ is the wavenumber of the input radiation. Then we define a modified electric field amplitude $y$ :

$$
y(z)=E(z) e^{i \delta(z)}
$$

where $\delta$ is the ponderomotive phase shift due to wiggler errors, as defined by Eq. (1.1).

We assume a planar wiggler with the wiggler wavenumber $k_{w}=2 \pi / \lambda_{w}$ and wiggler parameter $K_{0} \equiv e \lambda_{w} B_{\max } / 2 \pi m c$, where $B_{\max }$ is the maximum magnetic field on axis. The electron beam is assumed to be monoenergetic with energy $\gamma_{0}$, and satisfies the resonance condition $k=k_{w}\left(1+K_{0}^{2}\right) / 2 \gamma_{0}^{2}$.

Now we introduce the Bonifacio's scaled wiggler distance ${ }^{[10]} \tau=2 \rho k_{w} z$, where $\rho$ is the Pierce parameter defined by

$$
\left(2 \rho \gamma_{0}\right)^{3}=e^{2} Z_{0} n_{0} K_{0}^{-2}[J J]^{2} / 4 m c k_{w}^{2},
$$

with $n_{0}$ the electron density, $Z_{0}=377 \Omega$ the vacuum impedance, and $[J J]$ the Bessel factor given by

$$
[J J]=J_{0}\left[\frac{K_{0}^{-2}}{4\left(1+K_{0}^{2} / 2\right)}\right]-J_{1}\left[\frac{K_{0}^{-2}}{4\left(1+K_{0}^{2} / 2\right)}\right] .
$$

With these definitions, we have obtained the FEL equation with wiggler errors $[9]$ :

$$
\frac{d^{3} y}{d \tau^{3}}-i y=i \frac{d^{2}}{d \tau^{2}}(f(\tau) y)
$$

where $f=d \delta / d$ : is the phase changing rate due to wiggler errors. It is easy to verify that 


$$
f \equiv \frac{d \delta}{d \tau}=-\frac{\gamma_{0}^{2}\left(x^{\prime 2}-x_{0}^{\prime 2}\right)}{1+K_{0}^{2} / 2} \frac{1}{2 \rho}
$$

where $x^{\prime}$ and $x_{0}^{\prime}$ are the transverse velocities with and without wiggler errors respectively and where the prime stands for the derivative with respect to $z$.

Solving Eq. (2.5) by perturbation theory and keeping terms up to second order in $f$, we found that if the correlation length of the error function $f$ is shorter than the gain length, the power growth ratio can be explicitly expressed by the averaged square of the phase shift per gain length $W$, defined by Eq. (1.2). If the wiggler steering error is not corrected within a gain length, the angular kick produced at one position can influence the phase shift rate at a later position farther away than a gain length. In this case, the correlation length of the function $f$ is longer than the gain length. We will address this situation in Section 5. Here we assume that the cc relation length of $f$ is shorter than the gain length, then the power growth ratio at $\tau=T$ is:

$$
\frac{P_{\text {out }}}{P_{\text {in }}}=\frac{1}{9} e^{\left[\sqrt{3}+2 \overline{\Delta r_{g}} \pm 2 \delta\left(\Delta r_{g}\right)\right] T+c \pm \delta c}
$$

where

$$
\begin{gathered}
c=\frac{2 W \sqrt{3}}{27} \\
\delta c=\left(\frac{\sqrt{3} W}{9}\right) 1 / 2 \\
\overline{\Delta r_{g}}=-\frac{W}{9} \\
\delta\left(\Delta r_{g}\right)=\left(\overline{\Delta r_{g}}\right)\left(\frac{17 \sqrt{3}}{24 T}-\frac{49}{144 T^{2}}\right)^{1 / 2} .
\end{gathered}
$$

The fluctuations in the power come from two terms. The term $\delta c$ is the rms fluctuation of the coefficient of the growth factor, and is independent of the wiggler length. We found this term is determined by the errors at the beginning and the end of the wiggler. The term $\delta\left(\Delta r_{g}\right)$ is the rms fluctuation in the growth rate. Since $\delta c$ is proportional to $\sqrt{W}$. and is independent of $T$. while the average reduction in gain is proportional to IVT. for smaller wiggler error (and/or) shorter wiggler, the spread in the output power becomes comparable or larger than the average reduction in power. 


\section{Effect Due to Wiggling Amplitude Errors}

For wiggling amplitude errors, we have considered $[9]$ a model for which the two half periods of every period have the same errors but with opposite signs, so that there is no net steering effect, and there is only fluctuation in the wiggling amplitude. We have found:

$$
W=\frac{\pi K_{0}^{4}}{\left(1+K_{0}^{2} / 2\right)^{2}} \frac{(\Delta B / B)_{r m s}^{2}}{\rho},
$$

It follows that the criterion for small gain reduction is

$$
\frac{2 \pi}{9 \sqrt{3}} \frac{K_{0}^{4}}{\left(1+K_{0}^{2} / 2\right)^{2}} \frac{(\Delta B / B)_{r m s}^{2}}{\rho} \ll 1 .
$$

There is a simple physical interpretation of Eq. (3.1). Since the transverse wiggling velocity is $x^{\prime}=\left(K_{0}+\delta K\right) / \gamma_{0}$, where $\delta K$ is the increment of $K$ due to $\Delta B$, using the expression Eq. (2.6) for the phase shift rate, we get the change $\delta_{p}$ in ponderomotive phase per wiggler period due to wiggling amplitude errors

$$
\delta_{p}=\frac{2 \pi K_{0}^{2}}{1+K_{0}^{2} / 2} \frac{\Delta B}{B} .
$$

The number of periods per gain length is approximately $N_{G}=1 / 4 \pi \rho$, hence

$$
W=N_{G}\left(\delta_{p}\right)_{r m s}^{2}
$$

in agreement with Eq. (3.1).

We have carried out a computer simulation of this model using the computer program TDA ${ }^{[4,13]}$. The transverse electron beam size is taken to be large enough so that the radiation field on axis evolves as in the one-dimensional limit. In particular, we considered the parameters $\rho=1.29 \times 10^{-3}, K_{0}=1.95$, and $\left(\Delta B / B_{m a x}\right)_{r m s}=2 \%$. From Eq. (3.1) it follows that $W=1.67$ and hence the results of our analytic calculation [Eqs. (2.8-2.11)] are:

$$
\begin{array}{cl}
2 \overline{\Delta r_{g}}=-0.37, \quad & 2 \delta\left(\Delta r_{g}\right)=0.41 / \sqrt{T} \\
c=0.11, & \delta c=0.40
\end{array}
$$


The computer simulation results are presented in Fig. 1. The ratio of the output to input power is shown as a function of distance for 16 sets of random wiggler errors, and good agreement is found with the analytic estimate of Eq. (2.7). In particular the analytic estimate: of the average reduction in gain, $2 \overline{\Delta r_{g}}$, agrees with the numerical results to a few percent. As mentioned earlier, the spread in the exponent consists of two contributions. One is the spread in the constant $\pm \delta c$ and the other is the spread in the slope $\pm 2 \delta\left(\Delta r_{g}\right)$. The simulation is consistent with the width being dominated by the spread in the constant $\pm \delta c$ as predicted by the analytical theory taking into account the statistics of a limited number (16) of samples.

A more accurate check on the spread of the output power is done by directly comparing a numerical solution of Eq. (2.5) for 4000 sets of random wiggler errors with Eq. (2.9) and Eq. (2.11), the agreement is good to within $10 \%[9]$.

\section{Steering Errors with Trajectory Correction}

To study the gain reduction due to wiggler steering errors when the trajectory is being corrected, we assume the two half periods of every one period are uncorrelated, and there is a net field error integral and an angular kick for every period. In addition, we assume position monitors and trims are at the same locations spaced by $N_{S}$ periods along the wiggler. The spacing is assumed to be shorter than a gain length. Trim strength of a given corrector is adjusted to center the beam at the following monitor. In this case we have found ${ }^{[9]}$ that the average value of the square of the ponderomotive phase shift per gain length is given by

$$
W \approx \frac{24}{35} \frac{N_{G}}{N_{S}}\left(N_{S} \delta_{p}\right)^{2}
$$

with the rms phase shift per period $\delta_{p}$ in this case easily seen to be given by

$$
\delta_{p}=\frac{2 \pi \gamma^{2} x_{r m s}^{\prime 2}}{1+\Pi_{0}^{-2} / 2},
$$

where the mean squared angular deviation $x_{r m s}^{\prime 2}$ for the trajectory corrected beam is

$$
x_{r m s}^{\prime 2}=\frac{1}{6} N_{C} \overline{\theta^{2}}
$$


Here, $N_{C}$ is the number of steering errors between correction stations and $\overline{\theta^{2}}$ is the mean squared angular deviation introduced by a single error.

The result of Eq. (4.1) also has a simple physical interpretation. One sees that $N_{S} \delta_{p}$ is the ponderomotive phase shift between two correction stations, because the phase drift is coherent between correctors. Since phase drifts for different sections are incoherent, the total phase drift per gain length is equal to the square root of the number of correction sections per gain length times $N_{S} \delta_{p}$.

As an example consider a wiggler with $K_{0}=\sqrt{2}$. We assume the number of periods per gain length is $N_{G}=100\left(\rho \approx 0.8 \times 10^{-3}\right)$, the number of periods between correction stations is $N_{S}=50$ and the number of steering error kicks between correction stations is $N_{C}=100$. We express the mean square angular deflection per kick as

$$
\gamma^{2} \overline{\theta^{2}}=4 K_{0}^{2}(\Delta B / B)_{r m s}^{2}
$$

Taking the achievable tolerance $(\Delta B / B)_{r m s}=5 \times 10^{-3}$, we find $W=0.37$ resulting in a modest $5 \%$ reduction in the growth rate.

\section{Steering Errors without Trajectory Correction}

As pointed out in Section 2, when there is no orbit correction, or the correction distance is longer than the gain length, the correlation length of the error phase shift is long range, and we can not express the gain reduction in terms of $W$. However, we can still find the averaged power growth ratio:

$$
\frac{P_{\text {out }}}{P_{\text {in }}}=\frac{1}{9} e^{\left[\sqrt{3}+\frac{2}{3} \overline{\Delta r_{g}}\right] T} .
$$

with a growt?. rate reduction $[9]$

$$
\overline{\Delta r_{g}}=-\frac{\sqrt{3}}{18}\left[\frac{\gamma_{0}^{2} N_{T} \overline{\theta^{2}}}{2 \rho\left(1+K^{2} / 2\right)}\right]^{2} .
$$

Notice that the growth rate reduction now depends on the wiggler distance through the number of kicks $N_{T}$, which is twice the number of periods for our model in Section 4 . Thus. the gain reduction is a cubic function of the wiggler length. The physical interpretation of 
Eq. (5.1) is simple and similar to the discussion of the interpretation of Eq. (4.1) in Section 4. In this case, the error phase drift is coherent throughout the entire wiggler, and hence is $N_{G} \delta_{p}$ where $N_{G}=1 / 4 \pi \rho$ and $\delta_{p}$ is given by Eq. (4.2) with now $x_{r m s}^{\prime 2}=N_{T} \overline{\theta^{2}}$.

Using the same example of Section 4 , but reducing the rms wiggler error from $2 \%$ to $0.1 \%$ and assuming no orbit correction, we compared the theory with simulation. In Fig. 2 , we show the result of an average of $\log \left(P_{\text {out }} / P_{\text {in }}\right)$ over 30 samples, indicating a good agreement for long wiggler length and before saturation. For short wiggler, the fluctuation is larger, and the statistics of 30 samples is not sufficiently accurate. The result shows a much more stringent tolerance requirement on wiggler errors without correction scheme.

We wish to thank D. Goodman and J. Wurtele for providing us with a version of the computer code TDA including effects of wiggler error.

\section{References}

[1] B.M. Kincaid, J. Opt. Soc. Am. B2, 1294 (1985).

[2] C.J. Elliot and B.D. McVey, Proc. Conf. on Undulator Magnets for Synchrotron Radiation and Free Electron Lasers, Trieste, Italy, 1987 (World Scientific, Singapore, 1987).

[3] H.D. Shay and E.T. Scharlemann, Nucl. Instrum. and Meth. A272, 601 (1988).

[4] D. Goodman and J. Wurtele, private communication.

[5] B.L. Bobbs, G. Rakowsky, P. Kennedy, R.A. Cover, and D. Slater, Nucl. Instrum. and Meth. A269, 574 (1990).

[6] E. Esarey, W. Marable and C.M. Tang, Nucl. Instrum. and Meth. A296, 423 (1990).

[7] E. Esarey, C.M. Tang and W. Marable, J. Appl. Phys. 67, 2210 (1990).

[8] A. Friedman, in Proc. 1991 IEEE Particle Accelerator Conf., San Francisco, CA.

[G] L.H. Yu, S. Krinsky, R.L. Gluckstern and J.B.J. van Zeijts, "The Effect of Wiggler Errors on FEL Gain", submitted to Phys. Rev. A, (1991)

[10] R. Bnnifacio, C. Pellegrini and L.M. Narducci, Opt. Commun. 50, 373 (1984).

[11] W.B. Colson, IEEE J. Quantum Electron. QE-17. 1417 (1981).

[12] N..M. Kroll. P.L. Morton, M.N. Rosenbluth, IEEE J. Quantum Electron. QE-17. 1436 (1981). 
[13] T.M. Tran and J.S. Wurtele, Comp. Phys. Commun. 54, 263 (1989).

\section{Figure Captions}

1. The ratio $P_{\text {out }} / P_{\text {in }}$ as a function of wiggler length for 16 sets of random wiggling amplitude errors.

2. Without trajectoi'y correction, the average value of $\log \left(P_{\text {out }} / P_{\text {in }}\right)$ over 30 sets of random wiggler steering errors as a function of wiggler length. 

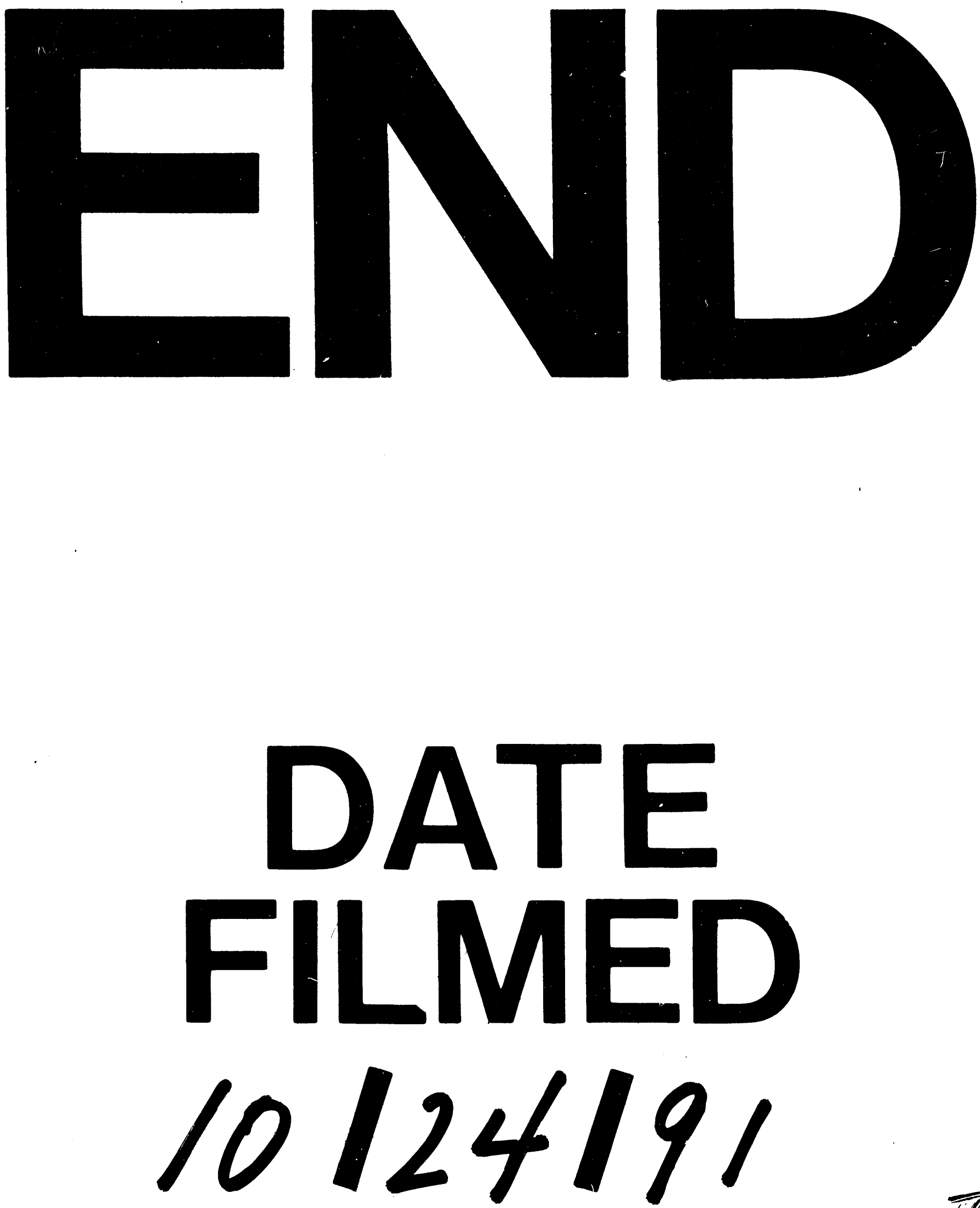
